\title{
Haptic Effects of Surgical Teleoperator Flexibility
}

\section{Citation}

Tavakoli, Madhi and Robert E. Howe. Forthcoming. International Journal of Robotics Research 28.

\section{Published Version}

http://www.ijrr.org/

\section{Permanent link}

http://nrs.harvard.edu/urn-3:HUL.InstRepos:2465625

\section{Terms of Use}

This article was downloaded from Harvard University's DASH repository, and is made available under the terms and conditions applicable to Other Posted Material, as set forth at http:// nrs.harvard.edu/urn-3:HUL.InstRepos:dash.current.terms-of-use\#LAA

\section{Share Your Story}

The Harvard community has made this article openly available.

Please share how this access benefits you. Submit a story.

Accessibility 


\title{
Haptic Effects of Surgical Teleoperator Flexibility
}

\author{
M. Tavakoli \\ Department of Electrical and Computer Engineering \\ University of Alberta \\ Edmonton, AB, Canada T6G 2V4 \\ tavakoli@ece.ualberta.ca
}

Robert D. Howe

BioRobotics Laboratory

School of Engineering and Applied Sciences

Harvard University

Cambridge, MA, USA 02138

howe@seas.harvard.edu

November 20, 2008 


\begin{abstract}
Minimally invasive surgery systems typically involve thin and cabledriven surgical instruments. This introduces link and joint flexibility in the slave robot of a master-slave teleoperation system, reducing the effective stiffness of the slave and the transparency of teleoperation. In this paper, we analyze transparency under slave link and joint flexibility (tool flexibility). We also evaluate the added benefits of using extra sensors at the tip of the flexible robot. It is shown that tip velocity (or position) feedback improves free-space position tracking performance in the presence of robot flexibility. Also, when the interaction forces with an environment are measured by a force sensor and fed back to the user's hand, tip velocity feedback improves hard-contact force tracking performance. During a hard contact task, tip velocity feedback can also eliminate the transmission of robot flexibility to the user's hand. ${ }^{1}$
\end{abstract}

\title{
Nomenclature
}

\author{
$\beta$ Control gain
}

$\omega_{0}$ Anti-resonance frequency

$\omega_{R}$ Resonance frequency

$C_{i}$ Controller

$C_{m}$ PD controller for master

$C_{s e} \mathrm{PD}$ controller for flexible slave (tip)

$C_{s m}$ PD controller for flexible slave (base)

\footnotetext{
${ }^{1}$ Parts of this research have previously been published as M. Tavakoli, R.D. Howe, The Effect of Joint Elasticity on Bilateral Teleoperation, In Proceedings of the 2007 IEEE/RSJ International Conference on Intelligent Robots \& Systems, pp. 1618-1623, San Diego, CA, 2007, and as M. Tavakoli, R.D. Howe, Haptic Implications of Tool Flexibility in Surgical Teleoperation, In Proceedings of the 16th Symposium on Haptic Interfaces for Virtual Environments \& Teleoperator Systems, pp. 377-378, Reno, NV, March 2008.
} 
$C_{s} \mathrm{PD}$ controller for rigid slave

$f_{e}, \tau_{e}$ Slave/environment interaction

$f_{h}, \tau_{h}$ Hand/master interaction

$f_{m}, \tau_{m}$ Controller output for master

$f_{s}, \tau_{s}$ Controller output for slave

$f_{12}$ Hard-contact force tracking metric

$f_{e}^{*}$ Environment's exogenous input force

$f_{h}^{*}$ Hand's exogenous input force

$H$ Hybrid matrix

$h_{11}$ Free-motion transmitted impedance

$h_{21}$ Free-motion position tracking metric

$k_{s}$ Slave stiffness

$M_{m}, I_{m}$ Inertia of master

$M_{s}, I_{s}$ Inertia of rigid slave

$M_{s e}$ Tip inertia of flexible slave

$M_{s m}$ Base inertia of flexible slave

$R M_{s e} / M_{s m}$

$R^{\prime} M_{m} / M_{s m}$

$v_{e}, \omega_{e}$ Slave tip (and environment) velocity

$v_{h}, \omega_{h}$ Master (and hand) velocity

$v_{h}^{*}$ Desired velocity for slave

$v_{s}, \omega_{s}$ Slave base velocity 
$x_{e}, \theta_{e}$ Slave tip (and environment) position

$x_{s}, \theta_{s}$ Slave base position

$z_{11}$ Hard-contact transmitted impedance

$Z_{e}$ Environment's impedance

$Z_{h}$ Hand's impedance

$Z_{s e}$ Tip impedance of flexible slave

$Z_{s m}$ Base impedance of flexible slave

$Z_{t}$ Impedance transmitted to the operator's hand

DFR Direct force reflection

PEB Position error based 


\section{Introduction}

In applications such as space and surgical robotics, it is advantageous to use thin and lightweight manipulators and cable-driven end-effectors. Space robots are designed to be lightweight and compact for minimum liftoff cost and energy consumption during robot control, and therefore involve flexibility. Surgical robots have thin instruments that enter the patient's body through ports for minimal invasiveness, which brings about advantages such as reduced trauma to the body, post-operative pain and length of hospital stay. An example is the Zeus Surgical Robot System (Figure 1) from Computer Motion Inc., Goleta, CA, USA, in which a $1 \mathrm{~N}$ force applied to the tip of one of its cantilevered instruments (straight endoscissors) causes a $15 \mathrm{~mm}$ tip deflection [Beasley and Howe, 2005]. As the surgical instruments become thinner (e.g., $<3 \mathrm{~mm}$ in pediatric surgery), the effect of flexibility becomes more crippling. Moreover, due to space limitations and the small diameter of the instruments in minimally invasive surgery, actuation of a distal wrist that is used for dexterity is performed from outside the patient and propagated to the wrist through flexible cables. Therefore, in addition to link flexibility, joint flexibility is often present in surgical robots. Besides flexibility, there are other non idealities that exist in practice and need to be accounted for including communication latency [Niemeyer and Slotine, 2004, Aziminejad et al., 2007, Pressman et al., 2007, Ohnishi and Mochizuki, 2007], encoder quantization [Abbott and Okamura, 2005], discrete-time implementation of haptic control laws [Gil et al., 2004, Love and Book, 1995, Tavakoli et al., 2007b], friction [Abbott and Okamura, 2005, Diolaiti et al., 2006], backlash, and noise.

In the presence of link or joint flexibility, control laws based on the assumption of a rigid robot may no longer be effective or accurate due to the alteration of the kinematic and dynamic characteristics of the manipulator. Without compensation, flexibility may cause steady-state errors, transient errors and vibrations, and even instability in the system. Dwivedy and Eberhard [Dwivedy and Eberhard, 2006] provided an extensive survey of the literature related to the dynamic analysis and control of flexible-joint and flexible-link 


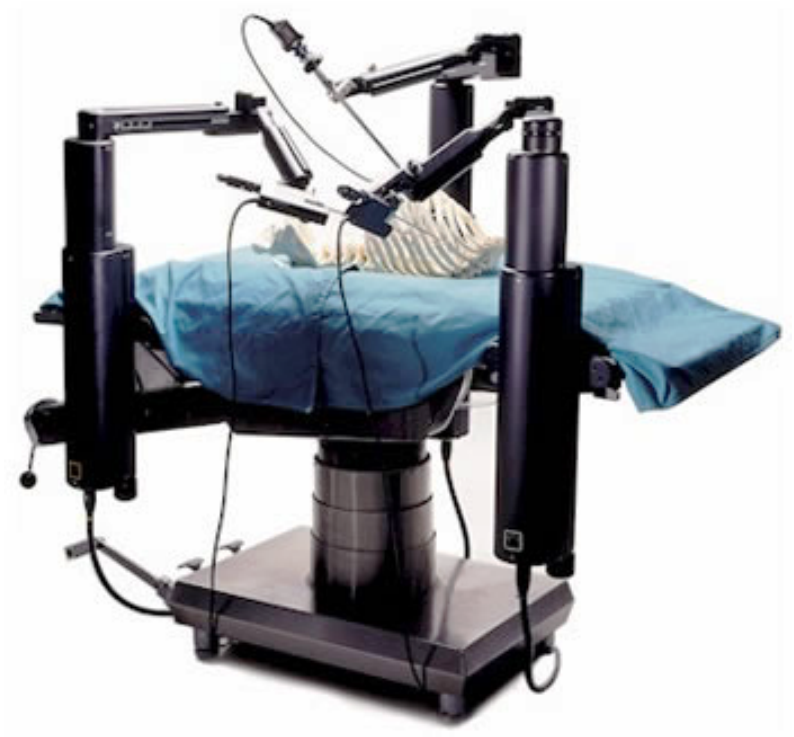

Figure 1: The Zeus Surgical Robot System.

robots. An example application in which joint flexibility needs to be compensated for is capturing non-cooperative objects such as space debris, where highbandwidth control is required [Nishida and Yoshikawa, 2003]. The pioneering work by Cannon and Schmitz [Cannon and Schmitz, 1984] pertained to the control of flexible-link robots when the sensors and actuators are not co-located. Beasley and Howe [Beasley and Howe, 2005] proposed a model-based method to reduce the kinematic errors in the control of a flexible surgical instrument.

Diaz and Gil [Diaz and Gil, 2008] analyzed the stability boundary of haptic rendering when the haptic user interface has internal vibration modes due to cable transmissions. For the specific problem of teleoperation with a low-stiffness slave, Christiansson and van der Helm [Christiansson and van der Helm, 2007] performed experiments with a 1-DOF master-slave system to demonstrate teleoperation performance/stability trade-offs. As metrics of performance, they measured the low-frequency asymptotes $\left(\lim _{s \rightarrow 0}\right)$ of the impedance transmitted to the operator when the slave is in free space and the impedance transmitted 
to the operator when the slave is in contact with a stiff environment. For quantifying a margin of stability, increasing amounts of delay were injected between the master and the slave until the teleoperation system approached the verge of instability. They concluded that the two performance metrics deteriorate in the presence of flexibility but this performance loss can be partly compensated for by incorporating deflection information in the control laws, and that the stability margin increases if the slave stiffness decreases.

In this paper, we systematically analyze performance and stability limitations under link or joint flexibility (tool flexibility) in the slave robot of a master-slave teleoperation system, and examine what added benefits tip sensors can deliver. This general analytical treatment considers the following four measures of performance: Free-motion transmitted impedance, free-motion position tracking, hard-contact force tracking metric, and hard-contact transmitted impedance. Two teleoperation architectures, position error based control and direct force reflection control, are considered mainly because of their implementation simplicity and widespread use. For both teleoperation control methods, we examine the effect of position and/or force sensors at the tip of a flexible slave on the above four performance metrics across the whole frequency range (i.e., for all $s$ ) and investigate the effect on the bandwidths of position and force tracking responses. We also conduct an analysis of absolute stability (stability under all passive but otherwise arbitrary human operators and remote environments) for the possible combinations of teleoperation methods and sensor configurations.

\section{Criteria for Analysis of Teleoperation Trans- parency and Stability}

For consistency with the teleoperation literature and without loss of generality, we use velocities rather than positions in models and control laws ${ }^{2}$. In an

\footnotetext{
${ }^{2}$ Note, however, the possibility of a steady-state error between the master and slave po-
} sitions when they have the same velocities. For an investigation of position drift in bilateral 
ideal 1-DOF master-slave teleoperation system with hand-master velocity $v_{h}$ and slave-environment velocity $v_{e}$, as in Figure $2 \mathrm{a}$, the dynamics of the master and the slave are

$$
f_{m}+f_{h}=M_{m} \dot{v}_{h}, \quad f_{s}-f_{e}=M_{s} \dot{v}_{e}
$$

where $f_{h}$ and $f_{e}$ denote the forces exerted by the operator's hand on the master and by the environment on the slave, respectively. $M_{m}, M_{s}, f_{m}$ and $f_{s}$ are the master and the slave inertias and control signals, respectively.

\section{$2.1 \quad$ Performance measures}

In an ideally transparent teleoperation system [Hannaford, 1989], through appropriate control outputs $f_{m}$ and $f_{s}$, the positions and contact forces at the master and the slave ends will match regardless of the operator and environment dynamics

$$
v_{h}=v_{e}, \quad f_{h}=f_{e}
$$

Condition (2) guarantees that the dynamics of the environment is displayed to the user with no distortion.

By considering Laplace transforms $V(s)$ and $F(s)$ of the velocities and forces in a teleoperation system, an equivalent representation of the system can be obtained [Hannaford, 1989] (Figure 2b), in which impedances $Z_{h}(s)$ and $Z_{e}(s)$ denote dynamic characteristics of the human operator's hand and the remote environment, respectively. Here, $F_{h}^{*}$ and $F_{e}^{*}$ are respectively the operator's and the environment's exogenous input forces and are independent of teleoperation system behavior. With the $s$-domain hybrid representation of a teleoperation system [Hannaford, 1989]

$$
\left[\begin{array}{c}
F_{h}(s) \\
-V_{e}(s)
\end{array}\right]=\left[\begin{array}{ll}
h_{11} & h_{12} \\
h_{21} & h_{22}
\end{array}\right]\left[\begin{array}{l}
V_{h}(s) \\
F_{e}(s)
\end{array}\right],
$$

teleoperation, see [Chopra et al., 2006, Ching and Book, 2006] 


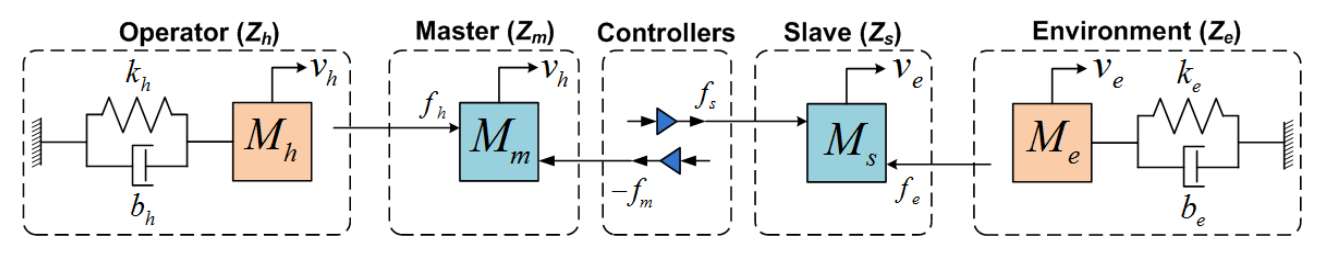

(a)

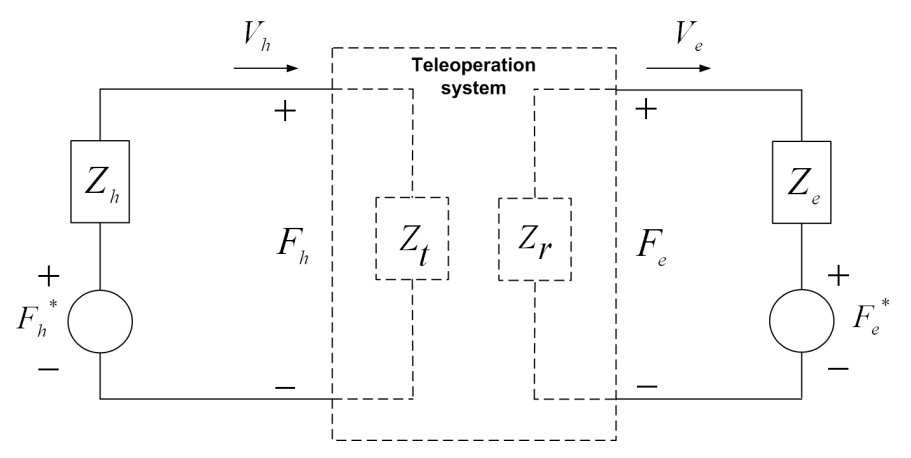

(b)

Figure 2: (a) Physical and (b) two port $s$-domain representations of a teleoperation system. 
(2) can be expressed as

$$
H_{\text {ideal }}=\left[\begin{array}{cc}
0 & 1 \\
-1 & 0
\end{array}\right]
$$

Limiting cases of two elements of the $H$ matrix

$$
h_{11}=\left.\frac{F_{h}}{V_{h}}\right|_{F_{e}=0}, \quad h_{21}=-\left.\frac{V_{e}}{V_{h}}\right|_{F_{e}=0}
$$

have direct physical significance. The parameter $h_{11}$ is the impedance transmitted to the user (input impedance) when $F_{e}=0$, i.e., the slave is in free space ( $\rightarrow 0$ ideally). Nonzero values for $h_{11}$ mean that the teleoperation system is providing the user with nonzero forces during free-motion movements. The parameter $h_{21}$ is a measure of velocity tracking fidelity when the slave is in free space $(\rightarrow-1$ ideally). Limiting cases of the other two parameters, i.e.,

$$
h_{12}=\left.\frac{F_{h}}{F_{e}}\right|_{V_{h}=0}, \quad h_{22}=-\left.\frac{V_{e}}{F_{e}}\right|_{V_{h}=0}
$$

are measures of force tracking fidelity and the output admittance assuming that the master is in contact with an infinitely stiff hand. Instead of $h_{12}$ and $h_{22}$, it is more useful to consider elements of the transmission and the impedance matrices [Aliaga et al., 2004]

$$
f_{12}=\left.\frac{F_{h}}{F_{e}}\right|_{V_{e}=0}, \quad z_{11}=\left.\frac{F_{h}}{V_{h}}\right|_{V_{e}=0}
$$

The above parameters assume that the slave is in hard contact. The parameter $f_{12}$ shows force tracking fidelity under hard contact ( $\rightarrow 1$ ideally) and the parameter $z_{11}$ is the maximum impedance that can be transmitted to the user ( $\rightarrow \infty$ ideally), thus quantifying the realism of a user's haptic experience about touching a rigid surface.

Colgate and Brown [Colgate and Brown, 1994] proposed using the $Z$-width, defined as $z_{11}-h_{11}$, as a measure of performance $(\rightarrow \infty$ ideally). An ideal haptic teleoperation system accurately reproduces both free motion and hard contact at the slave for the user. Another measure that is dependent on the hybrid parameters but provides important insight into the transparency of a 
teleoperation system is the environment impedance as transmitted to the user $\left(\rightarrow Z_{e}\right.$ ideally $)$

$$
Z_{t}=\frac{F_{h}}{V_{h}}=h_{11}-\frac{h_{12} h_{21} Z_{e}}{1+h_{22} Z_{e}}
$$

\subsection{Stability}

For analysis of stability of a teleoperation system, knowledge of the human operator and the environment dynamics are needed in addition to the teleoperation system model (3). However, assuming that $Z_{h}(s)$ and $Z_{e}(s)$ are passive, we may be able to find stability conditions independent of the human operator and the environment. The necessary and sufficient conditions for absolute stability (stability under all passive terminations $Z_{h}(s)$ and $Z_{e}(s)$ ) of a two-port network are given below.

\section{Llewellyn's criterion [Haykin, 1970]}

The two-port system (3) is absolutely stable iff: (a) $h_{11}(s)$ and $h_{22}(s)$ have no poles in the right half plane (RHP); (b) any poles of $h_{11}(\mathrm{~s})$ and $h_{22}(s)$ on the imaginary axis are simple with real and positive residues; and (c) for $s=j \omega$ and all real values of $\omega$

$$
\begin{aligned}
& \Re\left(h_{11}\right) \geq 0 \\
& \Re\left(h_{22}\right) \geq 0 \\
& 2 \Re\left(h_{11}\right) \Re\left(h_{22}\right)-\Re\left(h_{12} h_{21}\right)-\left|h_{12} h_{21}\right| \geq 0
\end{aligned}
$$

where $\Re(\cdot)$ and $|\cdot|$ show the real part and the absolute value.

\section{Teleoperator Model with Slave Flexibility}

The forgoing analysis tools are applicable to general models of the slave robot. We now consider the case when the slave has a flexible coupling between the actuator and the end-effector. 

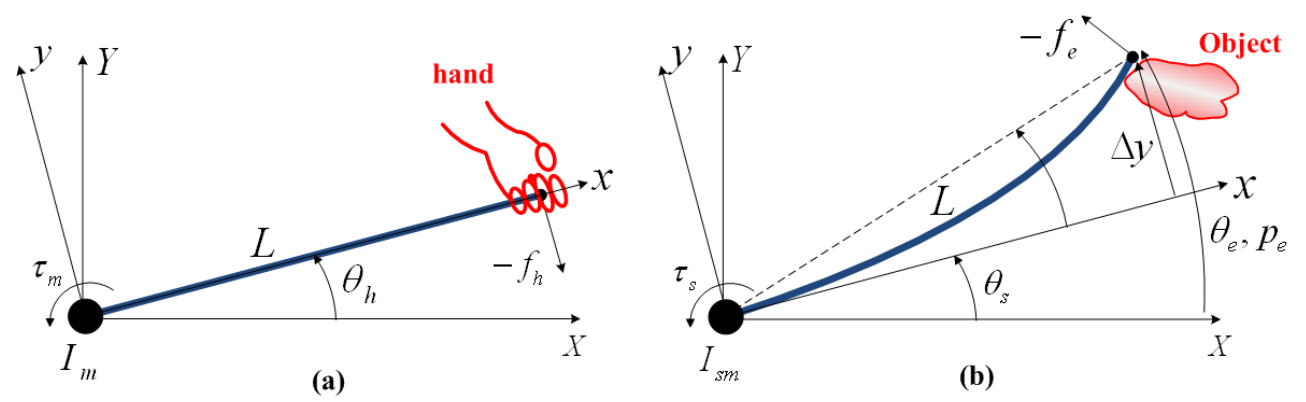

Figure 3: (a) The master; (b) the flexible-link slave.

\subsection{Case of a flexible-link slave}

An ideal 1-DOF teleoperation system, in which the master is rigid but the slave has a flexible tool that couples the actuator to the end-effector is shown in Figure 3, where $I_{m}, I_{s m}, \tau_{m}$ and $\tau_{s}$ are the master and the slave (excluding the flexible link) inertias and controller outputs, respectively. Also, $-f_{h}$ and $-f_{e}$ denote the forces exerted by the operator's hand on the master and by the environment on the slave, respectively. The hand-master position and the slave-environment position are denoted by $\theta_{h}$ and $\theta_{e}$ respectively, while $\theta_{s}$ is used to show the slave's joint position, which is different from $\theta_{e}$ due to the link flexibility. With a rigid link of length $L$ and defining $\omega_{h}=\dot{\theta}_{h}$ and $\tau_{h}=L f_{h}$, the dynamics of the master in Figure 3a are

$$
I_{m} \dot{\omega}_{h}=\tau_{m}+\tau_{h}
$$

The exact dynamics of a flexible link are described by partial differential equations and have infinite dimensions. In the constrained assumed modes method, the deflection of the flexible link in Figure $3 \mathrm{~b}$ is modeled as

$$
\Delta y(x, t)=\sum_{i=1}^{\infty} F_{i}(x) q_{i}(t), \quad 0 \leq x \leq L
$$

where $q_{i}(t)$ are the assumed flexible modes and $F_{i}(x)$ are the corresponding timeindependent modes shape functions. Considering the first mode $q_{1}(t)$, which is capable of capturing the dominant frequency, Zhu et al. [Zhu et al., 1999] 
presented a method for lumping the distributed mass of the flexible link to a point mass located at its tip followed by modeling the flexibility of the link by a massless linear bending spring. Denoting the equivalent tip lumped mass by $M_{s e}$ and the equivalent bending spring stiffness by $K_{s}$, the resulting lumped dynamic model of the flexible link in Figure $3 \mathrm{~b}$ is

$$
\begin{aligned}
M_{s e} \ddot{p}_{e} & =-K_{s} \Delta y-f_{e} \\
I_{s m} \ddot{\theta}_{s} & =\tau_{s}+L K_{s} \Delta y
\end{aligned}
$$

where $p_{e}=L \theta_{s}+\Delta y$ is the arc approximation of the link tip position assuming that $\Delta y$ is small. Noting that $\theta_{e}=p_{e} / L$ and defining

$$
\begin{aligned}
\Delta \theta & =\theta_{s}-\theta_{e}=-\Delta y / L \\
I_{s e} & =M_{s e} L^{2} \\
k_{s} & =K_{s} L^{2} \\
\tau_{e} & =L f_{e}
\end{aligned}
$$

the lumped model (14)-(15) of the flexible-link slave in Figure 3b is rewritten as

$$
\begin{aligned}
I_{s e} \dot{\omega}_{e} & =k_{s} \Delta \theta-\tau_{e} \\
I_{s m} \dot{\omega}_{s} & =\tau_{s}-k_{s} \Delta \theta
\end{aligned}
$$

where $\omega_{e}=\dot{\theta}_{e}$ and $\omega_{s}=\dot{\theta}_{s}$.

Interestingly, the lumped dynamics (20)-(21) of the flexible link are identical to the dynamics of the flexible joint shown in Figure 4 consisting of a motor with inertia $I_{s m}$ and an end-effector with inertia $I_{s e}$ that are coupled via a shaft with a finite stiffness $k_{s}$. Therefore, a flexible-link slave affects teleoperation performance in the same way as an elastic-joint slave.

\subsection{Case of a flexible-joint slave}

The compliance in the joint of a robot can be modeled by a chained massspring-damper system, in which the first mass represents the joint motor whose 


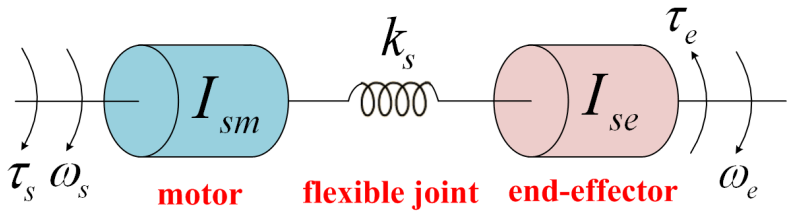

Figure 4: Model of a flexible joint.

position is measured and the last mass represents the end-effector by which the robot makes contact with the environment [Spong, 1987, Mills, 1992]. Figure 4 shows a rotational two-mass model with a spring. As it will be explained later, since we will be using a proportional-derivative $(\mathrm{PD})$ position controller for the flexible robot, there is no need to include a damper in the model because such a damping term would contribute to the closed-loop equation in the same way as the derivative term of the PD controller. In this model, $\tau_{s}$ and $\omega_{s}$ are the slave's motor torque and speed, respectively. Also, $\omega_{e}$ is the slave's end-effector (and the environment's) speed and $\tau_{e}$ is the torque applied by the environment on the slave's end-effector.

In the context of teleoperation control under slave joint compliance, we are interested in control of the slave's end-effector position, which is different from the motor position at least in the transient state, thus position sensing at the end-effector is useful. Depending on the teleoperation architecture and for better performance, we may also need force sensing at the end-effector.

For compatibility with the common notations in the teleoperation literature, we use the equivalent translational model of the elastic joint in the rest of this paper including in Figure 5, which shows a master-slave system with an elasticjoint slave. The equations of motion of the elastic joint present in Figure 5 are

$$
\begin{aligned}
M_{s m} \dot{v}_{s} & =f_{s}-k_{s} \Delta x \\
M_{s e} \dot{v}_{e} & =-f_{e}+k_{s} \Delta x \\
\Delta x & =x_{s}-x_{e}
\end{aligned}
$$




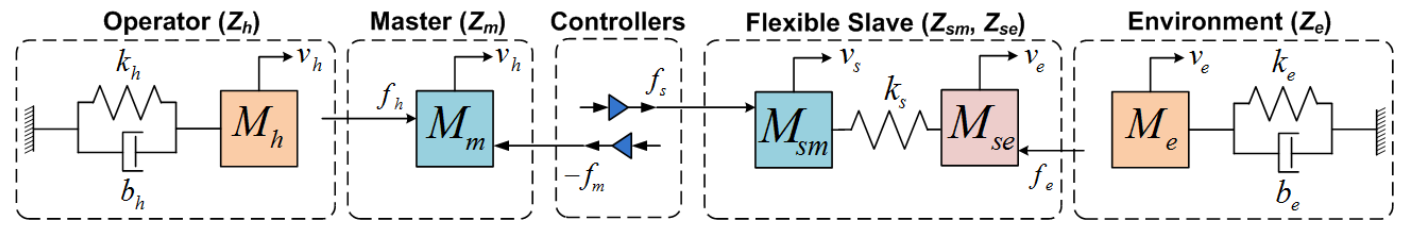

Figure 5: Models of the operator, master, flexible slave, and environment.

where $v_{s}=\dot{x}_{s}$ and $v_{e}=\dot{x}_{e}$ are the slave's motor and end-effector velocities, respectively. Also, $f_{s}$ is the force exerted by the slave's actuator on the elastic joint and $f_{e}$ is exerted by the environment on the slave's end-effector. An $s$ domain model of this two-input/two-output system is depicted in Figure 6c, in which $Z_{s m}=M_{s m} s$ and $Z_{s e}=M_{s e} s$.

Damping terms have not been considered in the master and the slave dynamics because such terms contribute to the closed-loop equations in the same way as the derivative terms of the master and slave PD controllers $\left(C_{m}\right.$ and $C_{s}$ in (29)), and therefore do not need to be considered separately. Also, to avoid complexities resulting from nonlinear terms, we have not considered backlash or friction in this analysis. The master and slave robot actuators are assumed to have unlimited bandwidths compared to the maximum frequency of the desired operating trajectories.

A state-space model of the two-mass system is

$\frac{\mathrm{d}}{\mathrm{d} t}\left(\begin{array}{c}v_{s} \\ \Delta x \\ v_{e}\end{array}\right)=\left(\begin{array}{ccc}0 & -\frac{k_{s}}{M_{s m}} & 0 \\ 1 & 0 & -1 \\ 0 & \frac{k_{s}}{M_{s e}} & 0\end{array}\right)\left(\begin{array}{c}v_{s} \\ \Delta x \\ v_{e}\end{array}\right)+\left(\begin{array}{c}\frac{1}{M_{s m}} \\ 0 \\ 0\end{array}\right) f_{s}+\left(\begin{array}{c}0 \\ 0 \\ \frac{-1}{M_{s e}}\end{array}\right) f_{e}$

The above system is state-controllable, meaning that if all states $\left(v_{s}, \Delta x\right.$ and $v_{e}$ ) are measurable, the eigenvalues of the system can be relocated to stable positions via state feedback.

The system (25) has one eigenvalue at the origin of the $s$-plane and two eigenvalues at $\pm j \omega_{R}$ where

$$
\omega_{R}=\sqrt{k_{s}\left(\frac{1}{M_{s m}}+\frac{1}{M_{s e}}\right)}
$$


is the system resonance frequency. For the control input $f_{s}$, if $v_{s}$ is the output, the system will have two zeros at $\pm j \omega_{0}$ where

$$
\omega_{0}=\sqrt{\frac{k_{s}}{M_{s e}}}
$$

is the system anti-resonance frequency. If $v_{e}$ is taken as the output, however, the system will show no anti-resonant behavior.

In the context of vibration control of steel rolling mills, which also suffer from flexibility due to the long shafts and gear boxes and have a model similar to $(25)$, it has been shown that the inertia ratio

$$
R=\frac{M_{s e}}{M_{s m}}
$$

plays a key role in shaping the dynamic characteristics of the elastic-joint system. When $R \ll 1$ and there is only feedback of $v_{s}$, the system has been reported to show a severely underdamped behavior [Zhang and Furusho, 2000]. In this situation, although the oscillations in $v_{s}$ may be small, those in $v_{e}$ may be large. However, with feedback of end-effector velocity $v_{e}$, it is possible to dampen such oscillations. In the following section, we examine the effect of joint flexibility in a robot that is acting as the slave during haptic teleoperation.

\section{Teleoperation Architectures vs. Sensor Con- figurations}

We now consider the relationship between the choice of the teleoperation control architecture [Tavakoli et al., 2007a] and the placement of sensors in a flexible slave. For a teleoperation architecture, different sensor configurations are possible. A velocity sensor on the slave's base (i.e., $v_{s}$ feedback), a velocity sensor at the end-effector (i.e., $v_{e}$ feedback), a force sensor at the end-effector (i.e., $f_{e}$ feedback), or a combination of them makes up the different possibilities.

Consider the block diagrams in Figure 6, which represent two common teleoperation control architectures and in which $C_{i}(s)$ are controller transfer functions. Position error based (PEB) bilateral control shown in Figures 6a uses no 
force sensor measurements and merely tries to minimize the difference between the master and the slave positions for providing haptic feedback to the user. Direct force reflection (DFR) bilateral control shown in Figures 6b, however, employs a force sensor to measure slave-environment interactions for reflecting them to the user.

We assume that the environment is passive $\left(f_{e}^{*}=0\right.$ in Figures $6 \mathrm{a}$ and $\left.\mathrm{b}\right)$ and the operator is passive in the sense that he/she does not perform actions that will make the teleoperation system unstable. In Figures $6 \mathrm{a}$ and $\mathrm{b}$, the human operator's hand and the remote environment impedances are denoted by $Z_{h}(s)$ and $Z_{e}(s)$, respectively. Also, $C_{1}, C_{4}$, and

$$
C_{m}=k_{p_{m}}+k_{i_{m}} / s, \quad C_{s}=k_{p_{s}}+k_{i_{s}} / s
$$

are controllers (PI-type on velocities and PD-type on positions). The gain $C_{2}$ scales the slave/environment interaction as it is fed back to the master. In Figures $6 \mathrm{a}$ and $\mathrm{b}$, based on (12), the master is represented as the impedance $Z_{m}^{-1}=1 /\left(M_{m} s\right)$. If the slave is rigid as in (12), it is modeled by the impedance $Z_{s}^{-1}=1 /\left(M_{s} s\right)$. If the slave is flexible, the two-output model based on (22)-(24) and shown in Figure 6c is used.

\subsection{Case of a rigid slave}

Assuming a rigid slave, in the position-error based (PEB) control of Figure 6a we have $C_{1}=C_{s}$ and $C_{4}=-C_{m}$. Transparency can be improved by including "acceleration feedforward" terms, i.e., by choosing $C_{1}=Z_{s}+C_{s} \doteq Z_{c s}$ and $C_{4}=-Z_{m}-C_{m} \doteq-Z_{c m}$ :

$$
H=\left[\begin{array}{cc}
Z_{m}+C_{m} \frac{Z_{s}}{Z_{c s}} & \frac{C_{m}}{Z_{c s}} \\
-\frac{C_{s}}{Z_{c s}} & \frac{1}{Z_{c s}}
\end{array}\right], \quad H_{\text {acc. }}=\left[\begin{array}{cc}
0 & \frac{Z_{c m}}{Z_{c s}} \\
-1 & \frac{1}{Z_{c s}}
\end{array}\right]
$$

For simplicity, in this paper $C_{m}=C_{s}$ is chosen.

Similarly, for a rigid slave, in the direct force reflection (DFR) control of Figure $6 \mathrm{~b}$, we have $C_{1}=C_{s}$ and $C_{2}=1$. Again, transparency is improved by 
including acceleration feedforward $\left(C_{1}=Z_{c s}\right)$ :

$$
H=\left[\begin{array}{cc}
Z_{m} & 1 \\
-\frac{C_{s}}{Z_{c s}} & \frac{1}{Z_{c s}}
\end{array}\right], \quad H_{\text {acc. }}=\left[\begin{array}{cc}
Z_{m} & 1 \\
-1 & \frac{1}{Z_{c s}}
\end{array}\right]
$$

\subsection{Case of a flexible slave}

In this case, since the flexible slave model has two outputs as shown in Figure 6c, $C_{s}$ and $C_{4}$ in Figures $6 \mathrm{a}$ and $\mathrm{b}$ are each broken into two separate controllers. Depending on the placement of sensors as discussed before, $C_{s}$ and $C_{4}$ should be replaced by either $C_{s m}$ or $C_{s e}$ and either $C_{4 m}$ or $C_{4 e}$.

The general control laws for the master in PEB and DFR architectures are defined as

$$
\begin{array}{ll}
F_{m}=-C_{m} V_{h}-\left(C_{4 m} V_{s}+C_{4 e} V_{e}\right) & \text { (Fig. 6a) } \\
F_{m}=-C_{2} F_{e} & \text { (Fig. 6b) }
\end{array}
$$

respectively. The control law for the slave in both PEB and DFR is defined as

$$
F_{s}=C_{1} V_{h}^{*}-\left(C_{s m} V_{s}+C_{s e} V_{e}\right) \quad(\text { Fig. 6a, b) }
$$

The desired slave velocity $v_{h}^{*}$ in (34) is normally equal to the operator's hand velocity $v_{h}$. However, with feedback of $v_{s}$ and $f_{e}$, it is possible to calculate $v_{h}^{*}$ more accurately based on the open-loop system equation (23). Taking the time

derivative of both sides of (23) gives $M_{s e} \ddot{v}_{e}=k_{s}\left(v_{s}-v_{e}\right)-\dot{f}_{e}$. Since $v_{e}=v_{h}$ is the performance goal, the estimated desired trajectory for $v_{s}$ becomes

$$
v_{h}^{*}=v_{h}+\left(M_{s e} / k_{s}\right) \ddot{v}_{h}+\dot{f}_{e} / k_{s}
$$

This estimate relies on derivatives of acceleration and force, which may be problematic to implement. We therefore consider teleoperator performance both with and without this estimate in Section 5 below. In the following section, we examine the effect of flexibility in a slave robot during haptic teleoperation. 

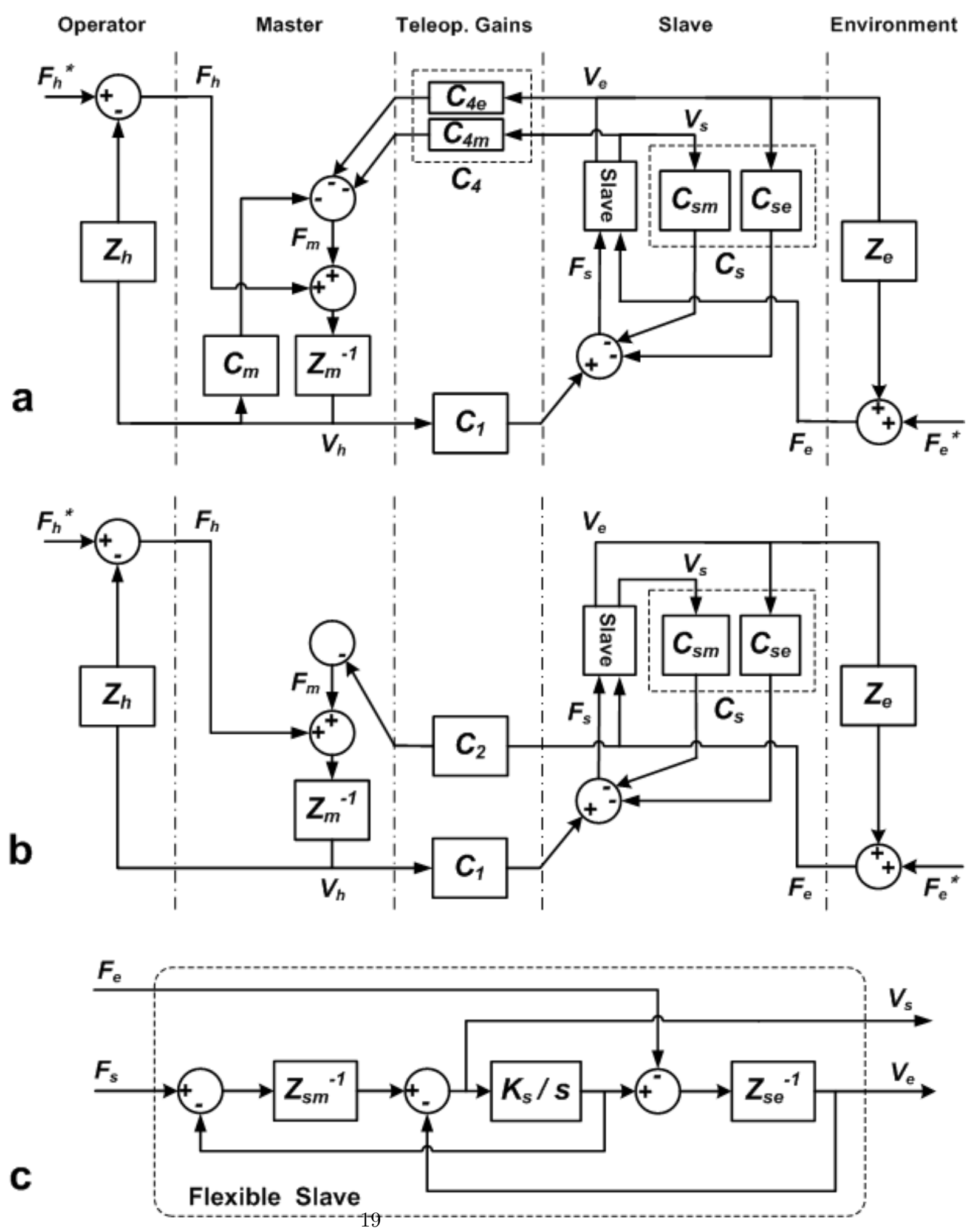

Figure 6: (a) Position error based (PEB) architecture, (b) direct force reflection (DFR) architecture, and (c) dynamic model of a flexible slave. 


\section{$5 \quad$ Performance measures}

\subsection{PEB control based on feedback of $v_{s}$}

With no feedback of $v_{e}$, we have $C_{s e}=C_{4 e}=0$ in Figure 6a. The PEB control with feedback of $v_{s}$ is designed as follows. We choose identical local PI controllers $C_{m}=C_{s m}=k_{p}+k_{i} / s$, and $C_{4 m}=-Z_{m}-C_{m}$. Since the flexible slave is comprised of two inertias, we consider the general form

$$
C_{1}=\eta_{1} Z_{s m}+\eta_{2} Z_{s e}+C_{s m}
$$

where $\eta_{1}$ and $\eta_{2}$ are non-negative constants. As a result, the parameter $h_{21}$ involves terms such as $k_{i}+k_{p} s+\eta_{1} M_{s m} s^{2}+\eta_{2} M_{s e} s^{2}$ in both its numerator and denominator, motivating the selections $\eta_{1}=1, \eta_{2}=0, k_{i}=\beta^{2} M_{s m}$ and $k_{p}=2 \beta M_{s m}$ (which ensure critical damping) where $\beta>0$ is a control gain determining the placement of poles in the system.

The resulting four measures of transparency defined in Section 2.1 are

$$
\begin{aligned}
h_{11} & =M_{s e} s \frac{1+\left(R^{\prime}-1\right)\left(\frac{s}{s+\beta}\right)^{2}}{1+\left(\frac{s}{\omega_{0}}\right)^{2}+R s^{2}\left(\frac{s}{s+\beta}\right)^{2}} \\
h_{21} & =\frac{-1}{1+\left(\frac{s}{\omega_{0}}\right)^{2}+R\left(\frac{s}{s+\beta}\right)^{2}} \\
\frac{1}{f_{12}} & =\frac{1}{1+\left(R^{\prime}-1\right)\left(\frac{s}{s+\beta}\right)^{2}} \\
z_{11} & =M_{s e} s \frac{1+\left(R^{\prime}-1\right)\left(\frac{s}{s+\beta}\right)^{2}}{\left(\frac{s}{\omega_{0}}\right)^{2}+R\left(\frac{s}{s+\beta}\right)^{2}}
\end{aligned}
$$

where

$$
R^{\prime}=\frac{M_{m}}{M_{s m}}
$$

and $\omega_{0}$ and $R$ have been defined in (27) and (28), respectively. The reason for using $1 / f_{12}$ is to have a proper transfer function because in the presence of flexibility the order of the numerator of $f_{12}$ increases. It can be seen that if $k_{s} \rightarrow \infty$ and $R \rightarrow 0$, the parameters corresponding to the rigid case (e.g., $h_{11}$ and $h_{21}$ of $H_{\text {acc. }}$ in (30)) will be retrieved.

Assuming the PI controller $C_{m}=C_{s m}$ does not become saturated, the control parameter $\beta$ can be selected to be sufficiently large so that the dynamics 
contributed by the controller (i.e., involving $\left.(s+\beta)^{2}\right)$ is much faster than the one originating from the rest of the system including joint flexibility. With this assumption, we get the simplified performance indices listed in the second column of Table 1.

While the above four parameters only depend on the teleoperation system, the impedance transmitted to the user is also a function of the environment impedance $Z_{e}$. Assuming a linear spring model $Z_{e}=k_{e} / s$ for the environment (i.e., $M_{e}=0$ and $b_{e}=0$ in Figure 5),

$$
Z_{t}=\frac{1 / s}{\frac{1}{k_{s}}+\frac{1 / k_{e}}{1+M_{s e} / k_{e} s^{2}}}
$$

The transmitted impedance represents the combined effect of $h_{11}$ and $z_{11}$. Evidently, when the slave is in free space $\left(k_{e} \rightarrow 0\right)$, we will have $Z_{t} \rightarrow h_{11}$, and when it is in contact with a hard environment $\left(k_{e} \rightarrow \infty\right)$, then $Z_{t} \rightarrow z_{11}$.

\subsection{PEB control based on feedback of $v_{e}$}

With feedback of $v_{e}$, we have $C_{s m}=C_{4 m}=0$ in Figure 6a. Again, $C_{m}=C_{s e}=$ $k_{p}+k_{i} / s, C_{4 e}=-Z_{m}-C_{m}$, and $C_{1}=\eta_{1} Z_{s m}+\eta_{2} Z_{s e}+C_{s e}$. For reasons similar to the case with feedback of $v_{s}$, we choose $\eta_{1}=1, \eta_{2}=0, k_{i}=\beta^{2} M_{s m}$ and $k_{p}=2 \beta M_{s m}$. The transparency indices for PEB control with feedback of $v_{e}$ when $\beta$ is sufficiently large are listed in the third column of Table 1.

\subsection{DFR control based on feedback of $v_{s}$ and $f_{e}$}

With feedback of $v_{s}$, we have $C_{s e}=0$ in Figure 6b. Also, $C_{s m}=k_{p}+k_{i} / s, C_{1}$ is chosen as in PEB control, and $C_{2}=1$. Depending on whether the desired $v_{s}$ is taken to be $v_{h}$ or calculated from (35), the simplified transparency indices for large $\beta$ are listed in the fourth and the fifth columns of Table 1, respectively.

\subsection{DFR control based on feedback of $v_{e}$ and $f_{e}$}

With feedback of $v_{e}$, we have $C_{s m}=0$ in Figure $6 \mathrm{~b}$. Also, $C_{2}=1$ and $C_{s e}=$ $k_{p}+k_{i} / s$. With $C_{1}$ chosen as in PEB control, the corresponding transparency 


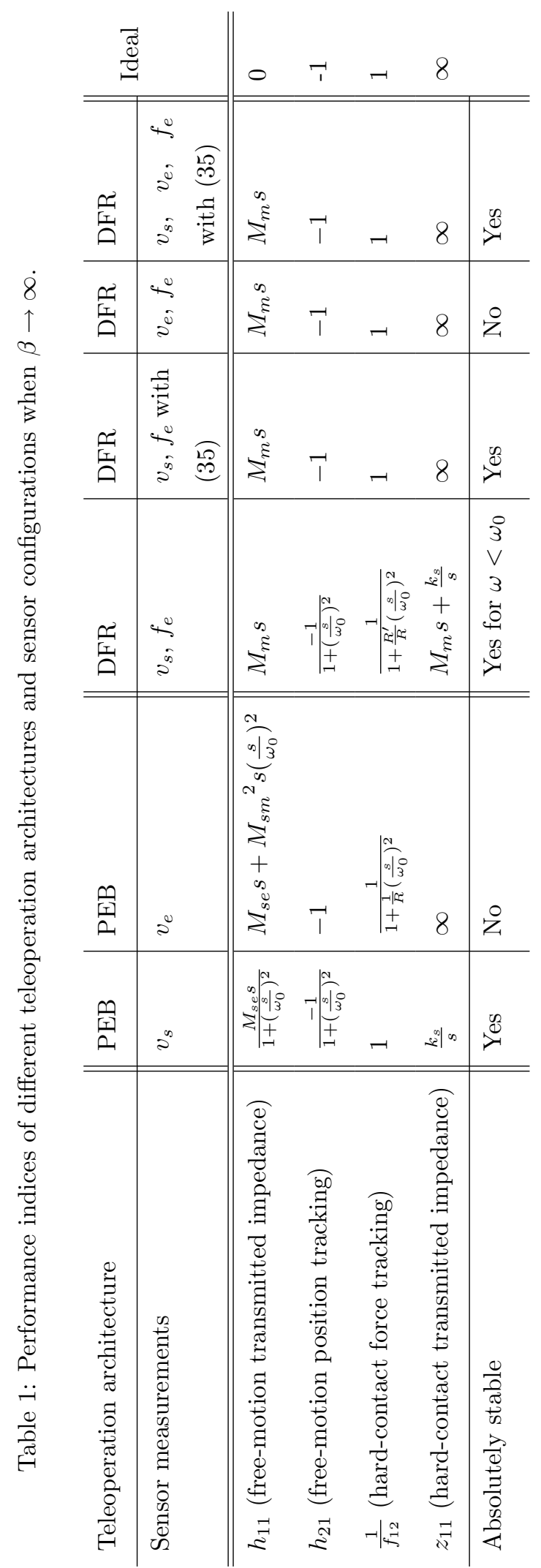


indices for large $\beta$ are listed in Table 1.

\subsection{DFR control with feedback of $v_{s}, v_{e}$ and $f_{e}$ :}

Since we have both $v_{s}$ and $v_{e}$ and we can also determine distinct desired trajectories for each of them due to the availability of $f_{e}$ information, we employ a two-loop PI controller as proposed in [Zhu et al., 1999] for the slave:

$$
F_{s}=\left(C_{1 m} V_{h}^{*}-C_{s m} V_{s}\right)+\left(C_{1 e} V_{h}-C_{s e} V_{e}\right)
$$

Here, $V_{h}^{*}$ is obtained from (35), $C_{1 m}=Z_{s m}+C_{s m}$ and $C_{1 e}=Z_{s e}+C_{s e}$.

\section{Effect of flexibility on Transparency and Sta- bility}

The transparency indices listed in Table 1 are idealized to ignore the effect of controller dynamics by assuming $\beta \rightarrow \infty$, which corresponds to perfect local position control of the slave (and the master during PEB control). This is a simplification that is made to isolate the effect of robot flexibility. With this assumption, Section 6.1 is aimed at understanding the fundamental limitations imposed by robot flexibility on teleoperation transparency and the added benefits of using extra sensors at the output shaft of an elastic-joint robot or at the tip of a flexible-link robot. In practice, however, $\beta$ cannot be infinitely large, thus bringing in the controller dynamics and limiting the performance. In Section 6.2 , we investigate the effect of limited control action on transparency, specifically the indices for motion tracking and force tracking.

\subsection{Transparency assuming no actuator saturation}

Based on Table 1 , which assumes $\beta$ is very large, the following conclusions can be drawn:

- For free-motion transmitted impedance ( $h_{11}$, third row), during PEB teleoperation the user will feel some residual impedance that depends on the 
slave's mass and stiffness characteristics, while during DFR teleoperation only the master inertia will be transmitted to the user. If acceleration feedforward were not provided during PEB, the user would feel the master inertia as well.

- For free-motion position tracking ( $h_{21}$, fourth row), with feedback of $v_{e}$, perfect position tracking can be attained in both PEB and DFR teleoperation regardless of the robot flexibility. With feedback of $v_{s}$, perfect position tracking in DFR teleoperation is possible if the desired trajec-

tory for $v_{s}$ is determined from (35). Otherwise, position tracking with $\omega_{s}$ feedback is satisfactory only at low frequencies $\left(\omega<\omega_{0}\right)$.

- For hard-contact force tracking $\left(1 / f_{12}\right.$, fifth row), perfect force tracking can be attained in PEB teleoperation with feedback of $v_{s}$. In DFR teleoperation, perfect force tracking is possible with feedback of $v_{e}$ and/or feedback of $v_{s}$ provided that (35) is used for generating the desired trajectory of $v_{s}$. Otherwise, force tracking is satisfactory only in low frequencies.

- For hard-contact transmitted impedance ( $z_{11}$, sixth row), with knowledge of $v_{s}$ only, the flexibility in the slave will be felt by the user during a hard contact task unless (35) is used for generating the desired trajectory of $v_{s}$. With feedback of $v_{e}$, however, hard surfaces can be displayed transparently to the user in both PEB and DFR teleoperation.

\subsection{Effect of avoiding actuator saturation on transparency}

Table 1 is accurate only for $\beta \rightarrow \infty$, but these relative performance characterizations for different teleoperation architectures and sensor configurations also apply for the case that $\beta$ is limited. Here we focus on the effect of $\beta$ on motion tracking and force tracking. It was shown that with feedback of $v_{e}$ and $\beta \rightarrow \infty$, it is possible to achieve ideal free-motion position tracking $\left(h_{21}=-1\right)$ regardless of the robot flexibility. This is not possible with feedback of $v_{s}$ even when $\beta \rightarrow \infty$ unless (35) is used. Also, ideal hard-contact force tracking $\left(1 / f_{12}=1\right)$ 
is possible with feedback of $v_{e}$ in DFR teleoperation, which is not attainable with feedback of $v_{s}$ even when $\beta \rightarrow \infty$ unless (35) is used. In the following we examine the effect of $\beta$ on $h_{21}$ and $1 / f_{12}$ for these two possible sensor configurations.

\subsubsection{Free-motion position tracking}

For teleoperation with feedback of $v_{s}, h_{21}$ (for both PEB and DFR) is given by

$$
\left.h_{21}\right|_{v_{s}}=\frac{-1}{1+\left(\frac{s}{\omega_{0}}\right)^{2}+R\left(\frac{s}{s+\beta}\right)^{2}}
$$

For teleoperation with feedback of $v_{e}$, the parameter $h_{21}$ (for both PEB and DFR) is given as

$$
\left.h_{21}\right|_{v_{e}}=\frac{-1}{1+\left(R+\left(\frac{s}{\omega_{0}}\right)^{2}\right)\left(\frac{s}{s+\beta}\right)^{2}}
$$

The magnitudes of the above two responses are plotted in Figure 7 when $\omega_{0}=$ $100 \mathrm{rad} / \mathrm{sec}, R=0.1$ and $\beta=20,100,1000$. For $v_{s}$ feedback, $\left.h_{21}\right|_{v_{s}} \approx 1$ only for frequencies lower than $\omega_{0}$ regardless of $\beta$. However, for $v_{e}$ feedback, we can increase the maximum frequency below which $\left.h_{21}\right|_{v_{e}} \approx 1$ by increasing $\beta$. Therefore, the frequency range of position tracking is improved if feedback of $v_{e}$ is provided and high-gain controllers are used. This is consistent with the discussion in Section 3.2 that the two-mass system (25) has an anti-resonance at $\omega_{0}$ if $v_{s}$ is the output but has no anti-resonance if $v_{e}$ is the output. Therefore, the presence of a velocity (or position) sensor at the output shaft of the elastic joint facilitates high-bandwidth position tracking during both PEB and DFR teleoperation.

To further investigate the effect of $\beta$ and $R$ on the shape of $h_{21}$, in Figure 8 the cutoff frequency $\omega_{c}$ (the frequency at which the magnitude drops by $-3 \mathrm{~dB}$ compared to low frequencies) of $h_{21}$ is plotted versus $\beta$ for $\omega_{0}=100 \mathrm{rad} / \mathrm{sec}$, $R=0.1,1,10$ and for feedback of $v_{s}$ (solid lines) and feedback of $v_{e}$ (dashed lines). As can be seen, with feedback of $v_{s}$, as $\beta \rightarrow \infty$ the cutoff frequency of $h_{21}$ given by (44) approaches $(\sqrt{2}+1)^{1 / 2} \omega_{0}=1.55 \omega_{0} \mathrm{rad} / \mathrm{sec}$. In contrast, with feedback of $v_{e}$, the cutoff frequency of $h_{21}$ given by (45) continues to grow as 


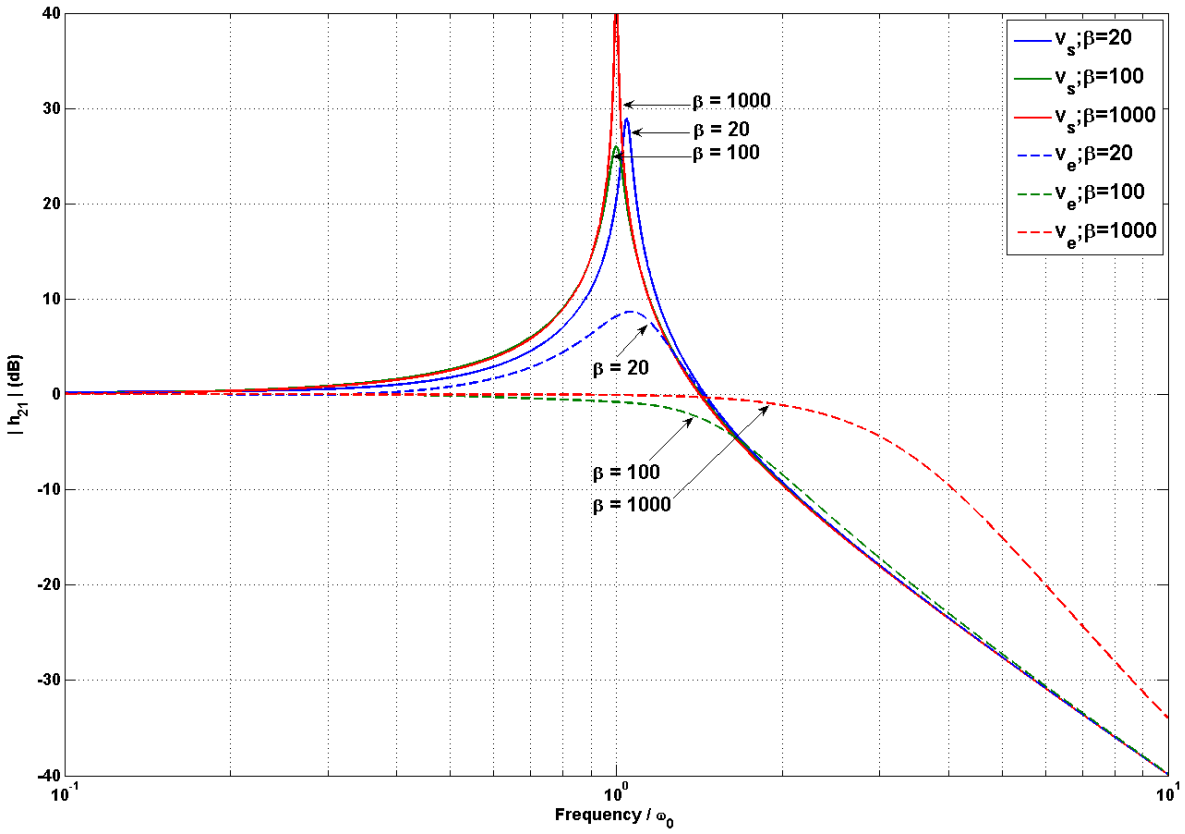

Figure 7: Magnitude of $h_{21}$ with feedback of $v_{s}$ (solid lines) and feedback of $v_{e}$ (dashed lines) vs. normalized frequency when $R=0.1$. 


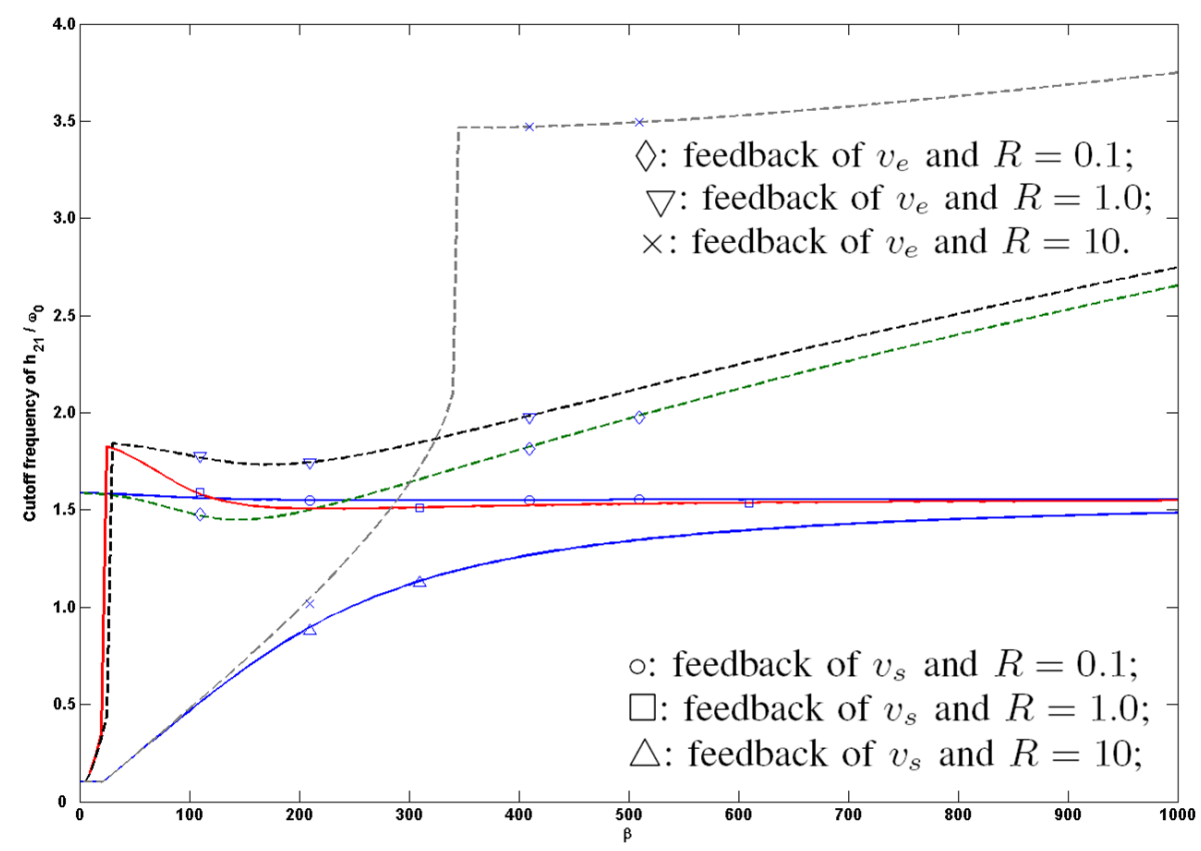

Figure 8: Cutoff frequency of $h_{21}$ with feedback of $v_{s}$ (solid lines) and feedback of $v_{e}$ (dashed lines) when $\omega_{0}=100 \mathrm{rad} / \mathrm{sec}$.

$\beta$ increases, thus ensuring good position tracking over a wider frequency range. The other conclusion from Figure 8 is that when $R$ is not small and $\beta$ is not large (i.e. $M_{s e}$ is comparable to or larger than $M_{s m}$ and the control effort applied on $M_{s m}$ is limited), the position tracking bandwidth is severely limited even with feedback of $v_{e}$ as the cutoff frequency of $h_{21}$ drops below $\omega_{0}$. Therefore, as $R$ gets larger, the need for higher control action (higher $\beta$ ) increases to attain satisfactory position tracking. 


\subsubsection{Hard-contact force tracking}

In DFR teleoperation, $1 / f_{12}$ for the two different position feedback possibilities is given by

$$
\begin{aligned}
\left.\frac{1}{f_{12}}\right|_{\mathrm{DFR}, v_{s}} & =\frac{1}{1+\frac{R^{\prime}}{R}\left(\frac{s}{\omega_{0}}\right)^{2}+R^{\prime}\left(\frac{s}{s+\beta}\right)^{2}} \\
\left.\frac{1}{f_{12}}\right|_{\mathrm{DFR}, v_{e}} & =\frac{1}{1+\frac{R^{\prime}}{R}\left(\frac{s}{\omega_{0}}\right)^{2}\left(\frac{s}{s+\beta}\right)^{2}+R^{\prime}\left(\frac{s}{s+\beta}\right)^{2}}
\end{aligned}
$$

Somewhat similar to the case with $h_{21}$, when we have feedback of $v_{s}$, near-ideal force tracking under hard-contact is obtained only for frequencies lower than

$\omega_{0} \sqrt{R / R^{\prime}}$ regardless of the maximum control effort. In contrast, with $v_{e}$, the cutoff frequency of $1 / f_{12}$ can be increased by increasing $\beta$. Therefore, in DFR teleoperation, feedback of $v_{e}$ also helps to achieve high-bandwidth force tracking. The magnitudes of $1 / f_{12}$ and the relationship between the cutoff frequency of $1 / f_{12}$ and $\beta$ are similar to those in Figures 7 and 8 for $h_{21}$, and are not shown here.

\subsection{Absolute stability assuming no actuator saturation}

From the last row of Table 1, obtained through examining Llewellyn's criterion for absolute stability, teleoperation with feedback of $v_{e}$ alone is not absolutely stable. This is consistent with Vukosavic and Stojic [Vukosavic and Stojic, 1998] that stability in servo drives similar to Figure 4 gets more difficult with load velocity feedback $\left(v_{e}\right)$ as the closed-loop system will encompass torsional resonance modes. Nonetheless, in practice, friction dissipates energy, which has a stabilizing effect [Diolaiti et al., 2006]. Also, upper bounds on the dynamic ranges of the environment and operator impedances that exist in practice result in relaxed absolute stability conditions [Hashtrudi-Zaad and Salcudean, 2001]. Even without taking friction or the limited dynamic range of environment and operator impedances into account, we have shown that teleoperation is absolutely stable with feedback of $v_{s}$ alone, or $v_{e}$ and $v_{s}$ while (35) is used for generating the desired trajectory of $v_{s}$. 
Note that the absolute stability results in Table 1 are valid only for very large $\beta$ - stability analysis for a limited $\beta$ is complex and remains as future work.

\section{Simulation Study}

In order to confirm the transparency results of Table 1, we simulated the PEB and DFR teleoperation control architectures in MATLAB and SimuLink (The MathWorks, Inc., Natick, MA, USA) using a variable-step, continuous-time ode23 solver. We chose $M_{m}=M_{s m}=1 \mathrm{~kg}, M_{s e}=0.1 \mathrm{~kg}, k_{s}=1000 \mathrm{~N} / \mathrm{m}$, and therefore $R=0.1, R^{\prime}=1$ and $\omega_{0}=100 \mathrm{rad} / \mathrm{sec}$. Also, the control parameter $\beta=10^{6}$ was chosen to be large as was assumed in Table 1 . The excitation input $f_{h}$ in Figure 5 consisted of the sum of a number of sinusoids evenly-spaced in the frequency domain from zero to $1000 \mathrm{rad} / \mathrm{sec}$. The reason for this choice is that a multi-sine signal demonstrates a rich and almost uniform spectrum over the frequency range of interest and is a highly persistent excitation (pe) as the sum of $n$ sinusoids is pe of an order not less than $2 n-2$ [Soderstrom and Stoica, 1989]. Free-motion and hard-contact tests were simulated for 20 seconds using $k_{e}=0$ and $k_{e}=10^{7} \mathrm{~N} / \mathrm{m}$, respectively. By applying spectral analysis (MATLAB function spa), $h_{11}$ and $h_{21}$ were estimated using the free-motion test data via (5), and $1 / f_{12}$ and $z_{11}$ were estimated using the hard-contact test data via (7). As an example, the estimated magnitudes of the performance indices for DFR teleoperation with feedback of $v_{s}$ and $f_{e}$ are shown in Figure 9 (dashed lines), which closely follow the idealized indices listed in the fouth column of Table 1 (solid lines).

\section{Discussion and Concluding Remarks}

The results of the above analysis as listed in Table 1 are mainly useful for understanding how extra sensors at the tip of a flexible slave robot can enhance transparency. With minimally invasive surgical robots as one of the candidates 

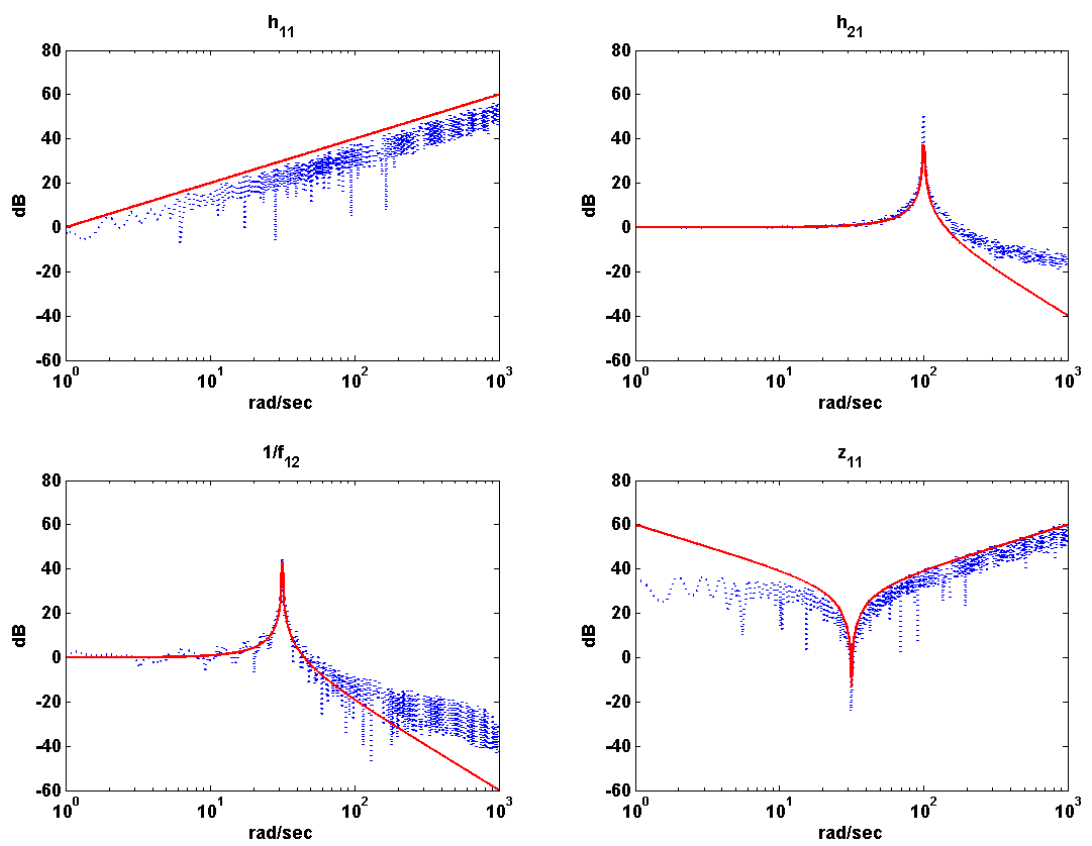

Figure 9: Magnitudes of the performance indices for DFR teleoperation for large $\beta$ with feedback of $v_{s}$ and $f_{e}$ when $R=0.1, R^{\prime}=1$ and $\omega_{0}=100 \mathrm{rad} / \mathrm{sec}$. Simulation results (dashed) and idealized models (solid). 
for which such an analysis is justified, however, arguments against adding sensors at the robot tip are made based on the fact that such sensors can complicate the design of the robotic arm, create sterilization issues, and ultimately raise the cost of the system. As a result, tip sensors have so far been avoided in today's commercial surgical systems (e.g., the da Vinci system from Intuitive Surgical Inc., Sunnyvale, CA, USA). On the other hand, in the specific example of the da Vinci robot, in order to avoid joint compliance, tensions in the cable drives are high. This has resulted in large friction in the instruments' drive trains, requiring sizable, remotely-based motors for tip actuation. In general, however, given the trade-off between joint compliance and friction in cable drives, joint compliance should be addressed separately especially in robots that are designed to be lightweight and cannot accommodate large actuators. Therefore, the questions addressed in this paper were, regardless of the state of sensor/actuator technologies in terms of meeting the requirements for integration in surgical or space robots, what are the limitations imposed by the slave robot flexibility on teleoperation transparency, what added benefits can tip sensors deliver during teleoperation with a flexible slave, and what are the cost-benefit tradeoffs of reducing or eliminating the effect of flexibility in haptic teleoperation?

- When the slave is in free space, unlike DFR teleoperation in which the user only feels the master inertia, in PEB teleoperation the user feels an additional impedance (which can be large - note the highly viscous term $s^{3}$ in the third column of Table 1). Such a residual impedance can create problems in terms of detecting small contacts or contact with very soft tissue, and a force sensor at the slave helps to avoid it.

- For both PEB and DFR teleoperation architectures, velocity (or position) feedback from the tip of the flexible slave improves free-space position tracking performance at higher frequencies, which is otherwise hampered by the anti-resonance of the two-mass-spring model unless (35) is used for generating the desired slave velocity. It is of practical interest to maintain good position tracking bandwidth in order to enable accurate and fast 
manipulation.

- In DFR teleoperation, tip velocity feedback or using (35) for calculating the desired slave velocity improves hard-contact force tracking performance. Otherwise, force tracking response will be band-limited, and the system will not be able to accurately simulate high-frequency haptic phenomena such as edges or surface texture of an object. Also, low-bandwidth haptic feedback has previously been shown to increases subjective workload in hard-contact assembly tasks.

- Over low frequencies, free-space position tracking and hard-contact force tracking are both satisfactory even in the absence of tip velocity feedback. However, in terms of the transmitted impedance, we showed that the only way to eliminate the display of robot flexibility to the user (even over low frequencies) is to either use tip velocity feedback or use (35) for generating the desired slave velocity. Previously, Christiansson and van der Helm [Christiansson and van der Helm, 2007] had concluded through experimental measurements with a low-stiffness slave that the maximum transmitted impedance can be doubled if tip velocity feedback is used in a 4-channel bilateral teleoperation architecture. Consistent with their results, we show that tip velocity feedback helps achieve an infinitely stiff transmitted impedance $\left(z_{11} \rightarrow \infty\right)$ in theory even with PEB and DFR teleoperation architectures, which have lower implementation complexity. The significance of this result is in the fact that if the robot flexibility is transmitted to the user, it will limit the perception of hitting a hard object (such as bone) and will make it more difficult to utilize haptic cues for soft-tissue stiffness discrimination. This has direct consequences, for example, in tissue palpation as a means to detect cancerous tissue, which has a different stiffness compared to healthy tissue.

Clearly, implementation issues are also important. While performance and stability benefit when (35) is used for generating the desired slave velocity, the 
trade-off is that obtaining low-noise velocity and force information for differentiation in (35) is problematic. Nevertheless, instead of using sensors to measure the end-effector velocity $v_{e}$ and the external force $f_{e}$, these quantities may be estimated using an extended state observer [Zhang and Tong, 2006] for less noisy signals. Evaluating the usefulness of such an observer remains as future work.

Looking beyond flexibility, backlash in the joints of a slave robot also has adverse effects on the transparency and stability of teleoperation. Surgical robots are a typical example of teleoperation systems with backlash due to the presence of cable-driven end-effectors. A similar analysis on the effect of backlash during bilateral teleoperation may quantify the problem and reveal ways to minimize it.

\section{Acknowledgements}

This research was supported by the National Science Foundation grant EEC9731748 and a postdoctoral fellowship awarded to the first author by the Natural Sciences and Engineering Research Council of Canada. The authors acknowledge the helpful discussions with Allison M. Okamura (Johns Hopkins Laboratory for Haptic Exploration) and Katherine J. Kuchenbecker (University of Pennsylvania GRASP Lab).

\section{References}

[Abbott and Okamura, 2005] Abbott, J. and Okamura, A. (2005). Effects of position quantization and sampling rate on virtual wall passivity. IEEE Transactions on Robotics, 21(5):952-964.

[Aliaga et al., 2004] Aliaga, I., Rubio, A., and Sanchez, E. (2004). Experimental quantitative comparison of different control architectures for masterslave teleoperation. IEEE Transactions on Control Systems Technology, 12(1):211. 
[Aziminejad et al., 2007] Aziminejad, A., Tavakoli, M., Patel, R. V., and Moallem, M. (2007). Wave-based time delay compensation in bilateral teleoperation: Two-channel versus four-channel architectures. In Proceedings of American Control Conference, pages 1449-1454, New York, NY.

[Beasley and Howe, 2005] Beasley, R. A. and Howe, R. D. (2005). Model-based error correction for flexible robotic surgical instruments. In Proceedings of Robotics: Science and Systems Conference, pages 359-364, Cambridge, MA.

[Cannon and Schmitz, 1984] Cannon, R. H. and Schmitz, E. (1984). Initial experiments on the end-point control of a flexible one-link robot. The International Journal of Robotics Research, 3(3):62-75.

[Ching and Book, 2006] Ching, H. and Book, W. (2006). Internet-based bilateral teleoperation based on wave variable with adaptive predictor and direct drift control. Journal of Dynamic Systems, Measurement, and Control, 128(1):86-93.

[Chopra et al., 2006] Chopra, N., Spong, M. W., Ortega, R., and Barabanov, N. E. (2006). On tracking performance in bilateral teleoperation. IEEE Transactions on Robotics, 22(4):861-866.

[Christiansson and van der Helm, 2007] Christiansson, G. A. V. and van der Helm, F. C. T. (2007). The low-stiffness teleoperator slave - a trade-off between stability and performance. The International Journal of Robotics Research, 26(3):287-299.

[Colgate and Brown, 1994] Colgate, J. E. and Brown, J. M. (1994). Factors affecting the $Z$-width of a haptic display. In IEEE International Conference on Robotics and Automation, pages 3205-3210, Los Alamitos, CA.

[Diaz and Gil, 2008] Diaz, I. and Gil, J. J. (2008). Influence of internal vibration modes on the stability of haptic rendering. In IEEE International Conference on Robotics and Automation, Pasadena, CA. 
[Diolaiti et al., 2006] Diolaiti, N., Niemeyer, G., Barbagli, F., and J.K. Salisbury, J. (2006). Stability of haptic rendering: Discretization, quantization, time delay, and coulomb effects. IEEE Transactions on Robotics, 22(2):256268.

[Dwivedy and Eberhard, 2006] Dwivedy, S. K. and Eberhard, P. (2006). Dynamic analysis of flexible manipulators, a literature review. Mechanism and Machine Theory, 41(7):749-777.

[Gil et al., 2004] Gil, J., Avello, A., Rubio, A., and Florez, J. (2004). Stability analysis of a 1 dof haptic interface using the routh-hurwitz criterion. IEEE Transactions on Control Systems Technology, 12(4):583-588.

[Hannaford, 1989] Hannaford, B. (1989). A design framework for teleoperators with kinesthetic feedback. IEEE Transactions on Robotics and Automation, $5: 426-434$.

[Hashtrudi-Zaad and Salcudean, 2001] Hashtrudi-Zaad, K. and Salcudean, S. E. (2001). Analysis of control architectures for teleoperation systems with impedance/admittance master and slave manipulators. Int. J. Robotics Research, 20(6):419-445.

[Haykin, 1970] Haykin, S. (1970). Active Network Theory. Addison-Wesley, Reading, MA.

[Love and Book, 1995] Love, L. and Book, W. (1995). Contact stability analysis of virtual walls. In Proceedings of the ASME International Mechanical Engineering Conference and Exposition, volume 57, pages 689-694.

[Mills, 1992] Mills, J. K. (1992). Stability and control of elastic-joint robotic manipulators during constrained-motion tasks. IEEE Transactions on Robotics, $8(1): 119-126$.

[Niemeyer and Slotine, 2004] Niemeyer, G. and Slotine, J. J. E. (2004). Telemanipulation with time delays. International Journal of Robotics Research, 23(9):873-890. 
[Nishida and Yoshikawa, 2003] Nishida, S. and Yoshikawa, T. (2003). Space debris capture by a joint compliance controlled robot. In Proceedings of IEEE/ASME International Conference on Advanced Intelligent Mechatronics, volume 1, pages 496-502, Kobe, Japan.

[Ohnishi and Mochizuki, 2007] Ohnishi, H. and Mochizuki, K. (2007). Effect of delay of feedback force on perception of elastic force: A psychophysical approach. IEICE Transactions on Communications, E90-B(1):12-20.

[Pressman et al., 2007] Pressman, A., Welty, L. J., Karniel, A., and MussaIvaldi, F. A. (2007). Perception of delayed stiffness. The International Journal of Robotics Research, 26(11-12):1191-1203.

[Soderstrom and Stoica, 1989] Soderstrom, T. and Stoica, P. (1989). System Identification. Prentice Hall International Ltd.

[Spong, 1987] Spong, M. W. (1987). Modeling and control of elastic joint robots. ASME Journal of Dynamic Systems, Measurement and Control, 109(4):310319.

[Tavakoli et al., 2007a] Tavakoli, M., Aziminejad, A., Patel, R., and Moallem, M. (2007a). High-fidelity bilateral teleoperation systems and the effect of multimodal haptics. IEEE Transactions on Systems, Man and Cybernetics Part B, 37(6):1512-1528.

[Tavakoli et al., 2007b] Tavakoli, M., Aziminejad, A., Patel, R. V., and Moallem, M. (2007b). Stability of discrete-time bilateral teleoperation control. In Proceedings of 2007 International Conference on Intelligent Robots and Systems, pages 1624-1630, San Diego, CA.

[Vukosavic and Stojic, 1998] Vukosavic, S. N. and Stojic, M. R. (1998). Suppression of torsional oscillations in a high-performance speedservo drive. IEEE Transactions on Industrial Electronics, 45(1):108-117. 
[Zhang and Furusho, 2000] Zhang, G. and Furusho, J. (2000). Speed control of two-inertia system by PI/PID control. IEEE Transactions on Industrial Electronics, 47(3):603-609.

[Zhang and Tong, 2006] Zhang, R. and Tong, C. (2006). Torsional vibration control of the main drive system of a rolling mill based on an extended state observer and linear quadratic control. Journal of Vibration and Control, 12(3):313-327.

[Zhu et al., 1999] Zhu, G., Ge, S. S., and Lee, T. H. (1999). Simulation studies of tip tracking control of a single-link flexible robot based on a lumped model. Robotica, 17(1):71-78. 